\title{
Fusion and Quasifission in Superheavy Element Synthesis
}

David J. Hinde

Department of Nuclear Physics, Research School of Physics and Engineering, The Australian National University, Canberra, ACT 2601, Australia

In the 1960s, quite soon after the recognition of the role of quantum shells in providing extra stability to nuclei, an "island of stability" of spherical superheavy nuclei was predicted. It was expected to be centred on proton number $Z=114$ and neutron number $\mathrm{N}=184$, the next magic numbers predicted above $\mathrm{Z}=82$ and $\mathrm{N}=126$ - which give nuclei around ${ }^{208} \mathrm{~Pb}$ their enhanced stability. Since then, intensive experimental efforts have been made to synthesise these superheavy elements - usually defined as those with atomic number 104 or more. These have been very successful, leading to the recent formal announcement of the discovery and naming of the last four new elements required to complete the $7^{\text {th }}$ row of the periodic table of the elements. These are nihonium $(Z=113)$, moscovium $(Z=115)$, tennessine $(Z=117)$, and the heaviest element yet discovered oganesson $(Z=118)$, named after Yuri Oganessian.

The mechanism of formation and the nuclear structure of superheavy elements (SHE) are key tests of quantum many body models, which are relevant to understanding neutron stars in astrophysics, and element formation and abundance in the Universe. Atom-by-atom measurements of superheavy element chemical properties are challenging, but allow testing of important relativistic effects in chemistry. Some of these measurements suggest that the periodicity of the chemical properties of elements with atomic number - the basis for Mendeleev's periodic table of the elements - may to be coming to an end.

The position and extent of the "island of stability" of superheavy nuclei is still not certain. Modern theoretical predictions of magic proton numbers in the superheavy region are in the range 114 to 126 . It seems likely that shell stabilization may occur across this region. This creates the possibility that isotopes of many new elements, heavier than those created up to now, will have lifetimes long enough to allow both nuclear and chemical properties to be experimentally determined. The problem then is how atoms of these elements can be synthesised.

The heaviest synthetic elements have all been created in collisions of two heavy nuclei, in which all the protons and neutrons of the colliding nuclei merge to form a new compact "compound" nucleus. Neutron evaporation from this excited compound nucleus competes with the predominant fission decay mode, occasionally resulting in the population of a superheavy nucleus in its ground-state. These are identified through a chain of characteristic decays. The sequence of six consecutive $\alpha$-decays within 47 seconds from copernicium-277 ( $Z=112)$ was described by superheavy element pioneer Peter Armbruster as "a poem of physics".

All the heaviest elements $(Z=114-118)$ have been formed in fusion reactions using beams of the doubly-magic neutron-rich nucleus ${ }^{48} \mathrm{Ca}(Z=20)$. Targets of elements heavier than $\mathrm{Cf}$ $(Z=98)$ cannot yet be made, as sufficient quantities of Es or Fm isotopes do not exist. To synthesise elements heavier than oganesson, heavier projectiles must therefore be used. Attempts to create $Z=119,120$ using heavier projectiles have not yet been successful, indicating that the production cross sections are smaller than when using beams of ${ }^{48} \mathrm{Ca}$. Thus, a key question in superheavy element research is how much smaller SHE cross sections will be when using beams of elements heavier than $\mathrm{Ca}$. To make reliable model predictions, up to $Z=126$, for example, the SHE formation process must be understood more thoroughly. 
The shell stabilization of these heaviest elements is strong only for near-spherical shapes the potential energy surface for more elongated shapes is repulsive. In fusion, the initial contact shape when two nuclei collide is elongated, being essentially that of the two touching nuclei. To create a SHE, this elongated shape must evolve to a compact nearspherical shape. However, this is resisted by the repulsive Coulomb-dominated potential. Consequently, a compact compound nucleus is rarely formed. Typically the initial kinetic energy is quickly damped, then the system performs a random walk over the repulsive potential energy surface, normally elongating and breaking apart into two heavy fragments. If the system breaks apart very quickly, there can be energy damping, but not enough time for significant mass evolution, and this is called a deep inelastic collision. If the system sticks together longer, the kinetic energy is fully damped, and some mass flow from the heavy to the light fragment occurs (as it moves towards more energetically favoured masssymmetric configurations) before it breaks apart. This non-equilibrium process is called quasifission [1,2]. It can result in a drastic suppression of the SHE formation cross section.

Quasifission can occur very rapidly [2,3], typically in less than $10^{-20} \mathrm{~s}$ (10 zeptoseconds). The probability of quasifission $\left(\mathrm{P}_{\mathrm{QF}}\right)$ can be very large, thus the complementary probability of fusion forming a compact compound nucleus $\left(\mathrm{P}_{\mathrm{CN}}=1-\mathrm{P}_{\mathrm{QF}}\right)$ can be small, probably lower than $10^{-3}$ in reactions forming the heaviest elements. Understanding and predicting the competition between quasifission and fusion is thus very important in mapping out the optimal fusion reactions to use in future to synthesize new elements and isotopes in the superheavy mass region. The most direct information on quasifission dynamics comes from measurements of the characteristics of the quasifission events themselves. Clearly, predictive models of superheavy element synthesis reactions should be able to describe measured quasifission characteristics (and how these change with different choices of colliding nuclei) as well as reproduce existing $P_{\mathrm{CN}}$ values determined though modelindependent measurements of heavy element formation cross sections $[4,5]$.

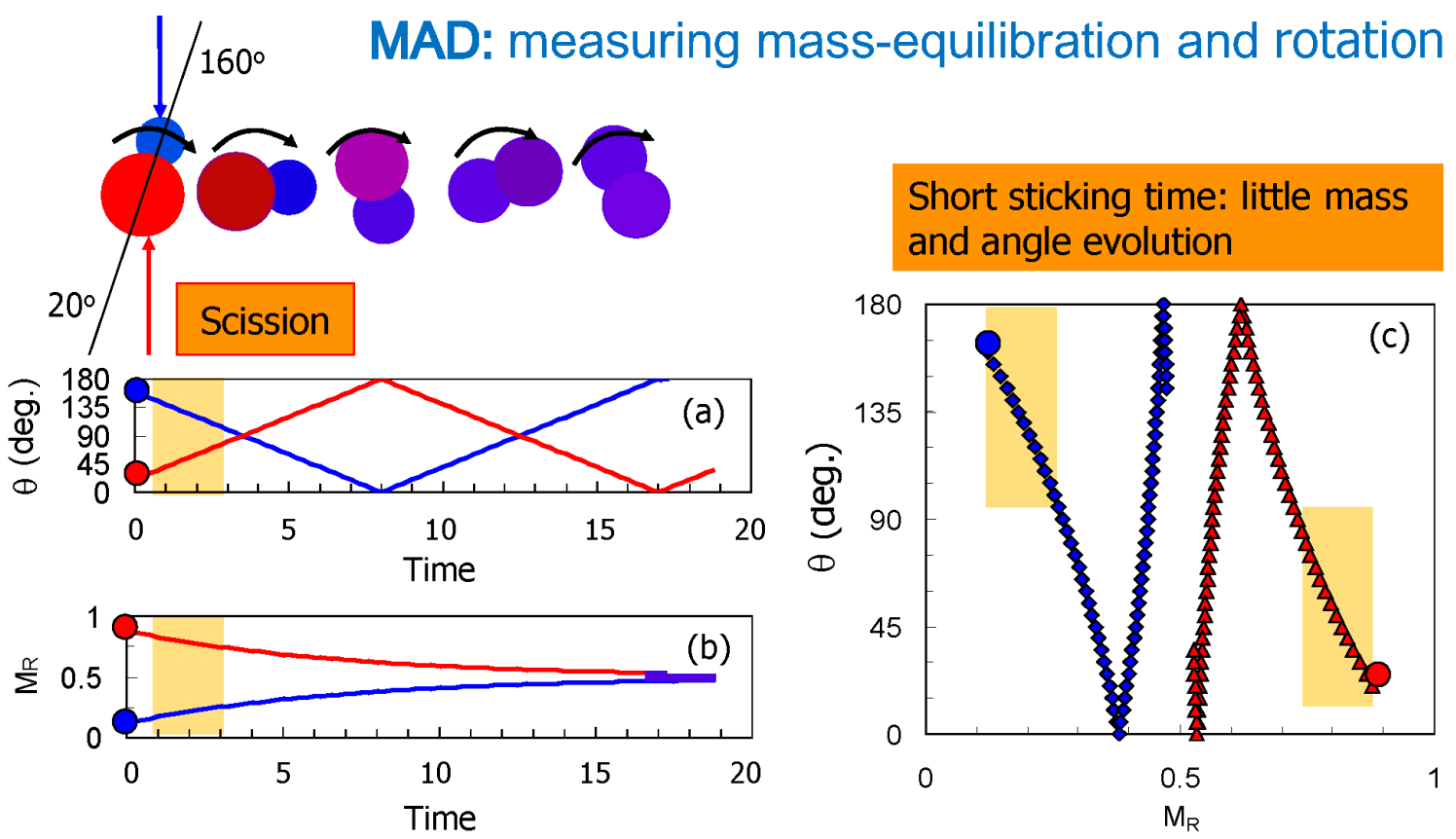

Figure 1.

The principles underlying the mass-angle distribution (MAD). The top left sketch illustrates the sequence of configuration changes after initial contact of light (blue) and heavy (red) nuclei, here with an initial centre-to-centre angle at contact of $160^{\circ}$. Panels (a) and (b) show schematically the rotation and mass-equilibration (in terms of mass-ratio $M_{R}$ - see text) as a function of time (in units of $10^{-21} \mathrm{~s}$ ). These zeptosecond times cannot be measured directly, however mass-ratio and angle can be measured. One trajectory in mass-ratio and angle is illustrated in (c), the orange highlighted region indicating the region of collision outcomes resulting from a short sticking time and rapid separation (scission). 
A key characteristic relevant for superheavy element formation is the "sticking time" following contact of the two nuclear surfaces. It is expected that the sticking time is correlated with $\mathrm{P}_{\mathrm{CN}}$ : where the sticking time is longer, then $\mathrm{P}_{\mathrm{CN}}$ would be expected to be larger (more favourable for SHE synthesis). The average sticking time can be inferred from measurements of the quasifission characteristics, as illustrated in Fig.1. The two colliding nuclei always approach each other along the beam axis, and after contact rotate with angular velocities that can be calculated [6]. Measurement of the rotation angle thus allows estimation of the sticking time. As the system rotates, mass flow also occurs between the two nuclei. Measurement of the velocity vectors of both fragments [2,7] provides excellent discrimination against fission of target-like nuclei resulting from peripheral (e.g. nucleon transfer) processes $[7,8]$, and furthermore allows determination of the centre-of-mass angle and mass-ratio $M_{R}=M_{1} /\left(M_{1}+M_{2}\right)$ of the fragments at scission (here $M_{1}, M_{2}$ are the fragment masses).

Measurements of $\mathrm{M}_{\mathrm{R}}$ over a wide range of angles is called a mass-angle distribution (MAD). This gives direct information on the dynamical time scales, as long as the system undergoes less than a full rotation (taking $\sim 10^{-20} \mathrm{~s}$ ). This is usually the case for collisions of heavy nuclei, as shown first by measurements at GSI [2], and later by results from ANU $[3,8,9,10$ and references therein $]$.
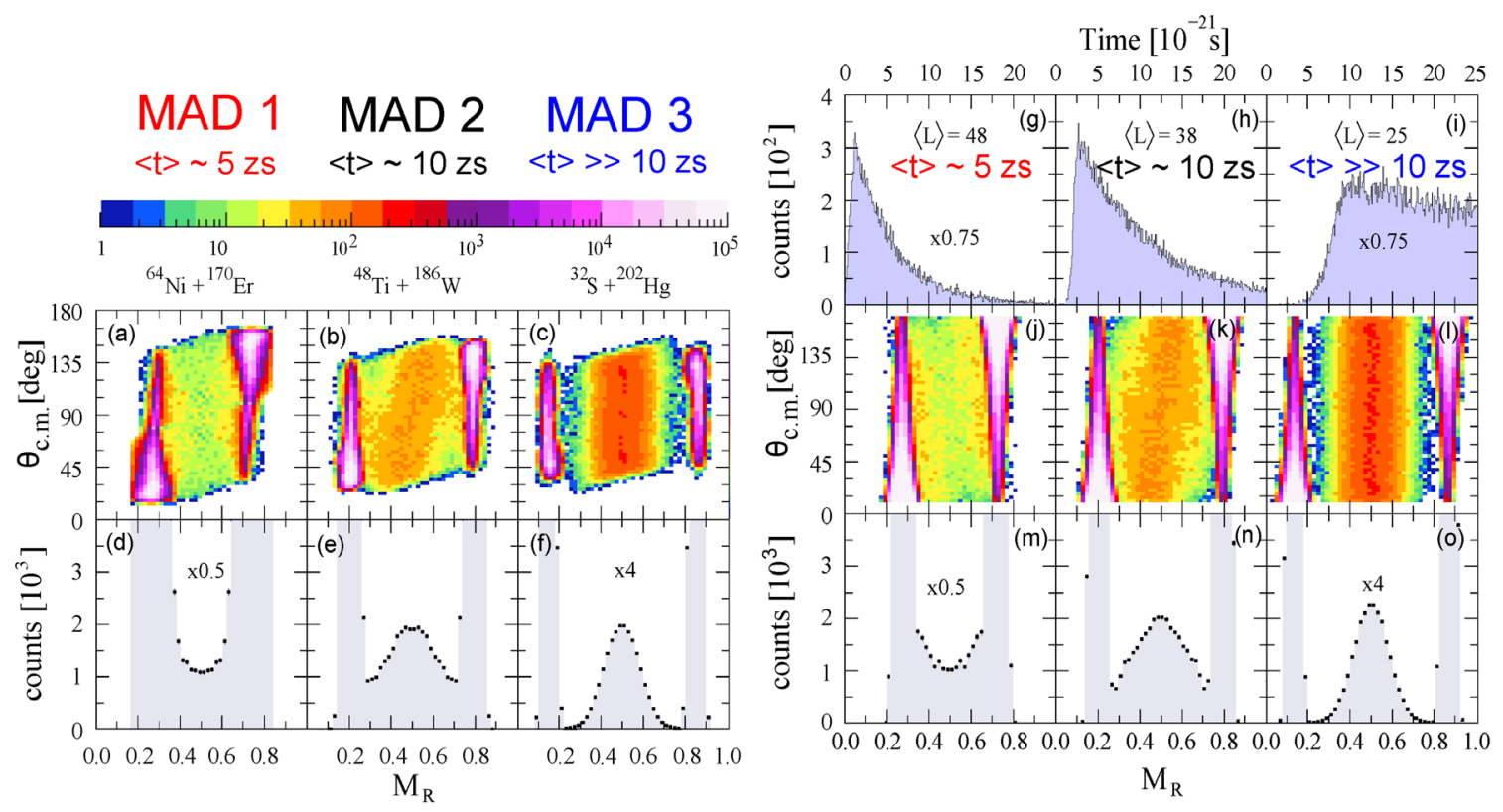

Figure 2

Panels (a)-(c) show experimental MADs, with (d)-(f) showing the projections onto $M_{R}$, for the indicated reactions which all form ${ }^{234} \mathrm{Cm}$. The multiplicative factors scale the y-axis. Simulated MADs for the same reactions and beam energies are shown in the right panels (j),(k),(l), with the corresponding $M_{R}$ spectra in panels $(m),(n),(o)$, and the sticking time distributions used in the simulations in $(g),(h),(i)$. The calculated mean angular momentum following capture, and the deduced approximate mean sticking times are also given (adapted from [9]).

Examples of measured MAD and deduced quasifission sticking time distributions are shown in Fig.2, for reactions forming the compound nucleus ${ }^{234} \mathrm{Cm}$ [9]. MAD can be most simply characterised [8] into three categories having: (i) a mass-angle correlation with a minimum yield at mass-symmetry - associated with short sticking times (MAD1, shown in Fig.2(a)); (ii) a mass-angle correlation with maximum yield at mass-symmetry - from intermediate sticking times (MAD2, shown in Fig.2(b)); and (iii) no significant mass-angle correlation and a narrow mass-distribution - associated with long sticking times, including fission following fusion (MAD3, shown in Fig.2(c)). 
The systematic trends of MAD characteristics with the identity of the two colliding nuclei was studied [8], to determine global trends of quasifission dynamics. This is in analogy with the evaluation of the liquid drop model dependence of nuclear masses on $\mathrm{N}$ and $\mathrm{Z}$, where deviations from smooth behaviour highlight the effects of nuclear structure. Choosing bombarding energies $\mathrm{E}$ well-above the mean capture barrier $\mathrm{B}$ (around $\mathrm{E} / \mathrm{B}=1.08$ ), nuclear structure effects were minimised. It was found [8] at these bombarding energies that the MAD are indeed strongly correlated with global variables. The simplest variables are the Coulomb repulsion in the entrance channel (related to the product of the proton numbers of the projectile and target nuclei $\mathrm{Z}_{\mathrm{p}} \mathrm{Z}_{\mathrm{t}}$ ), and the compound nucleus atomic number $Z_{\mathrm{CN}}$, as illustrated in Fig.3. However, for particular reactions, it has been found that the nuclear structure of the nuclei in the entrance channel can change the sticking times and MAD characteristics dramatically. This is clearly seen for doubly-magic neutron-rich nuclei such as ${ }^{48} \mathrm{Ca}$ and ${ }^{208} \mathrm{~Pb}$, and prolate deformed nuclei (all used in SHE formation reactions), as described below.

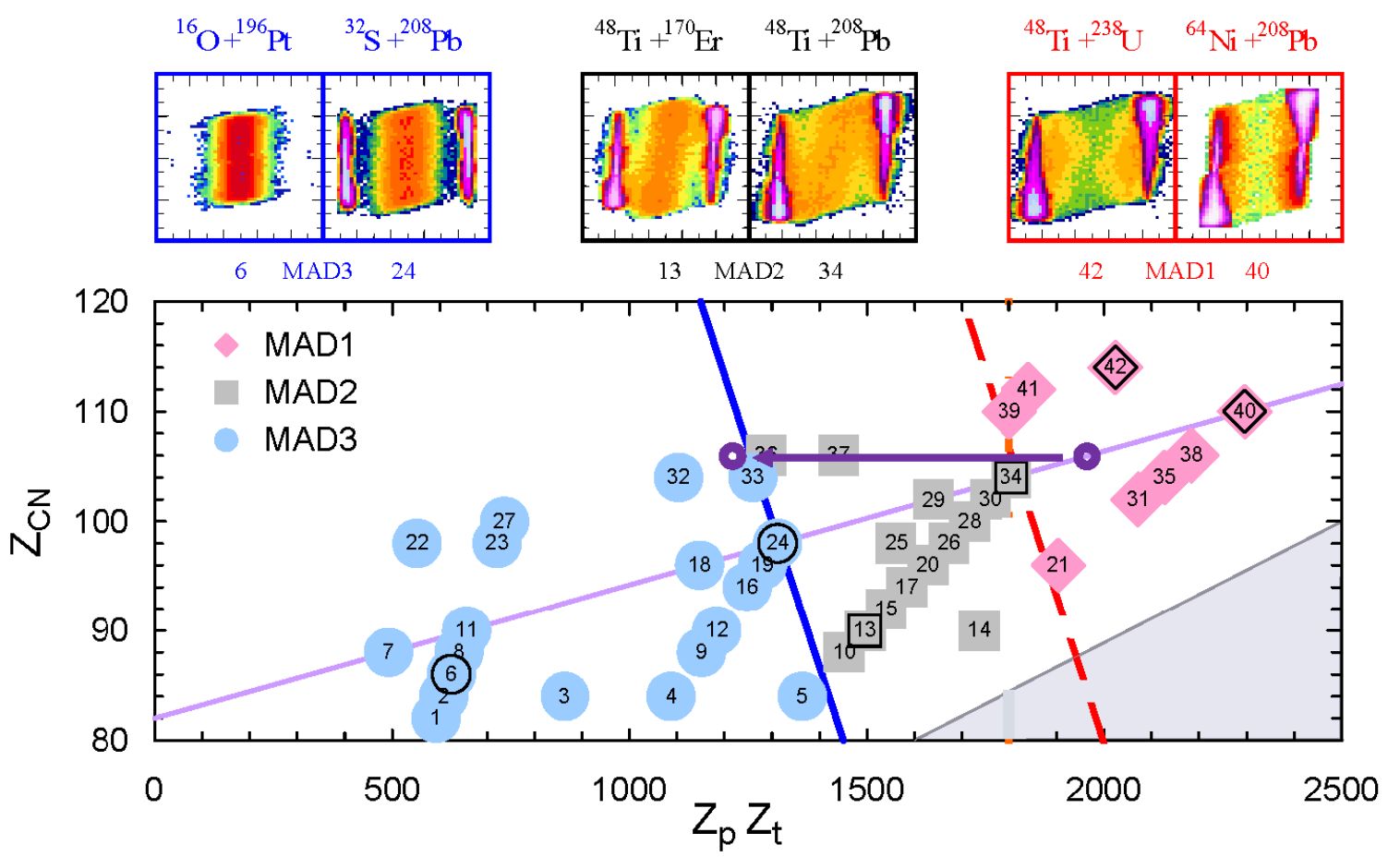

Figure 3

The symbol indicates the classification of MAD observed, shown as a function of the charge product of the colliding nuclei $Z_{p} Z_{t}$ and the atomic number of the compound nucleus $Z_{C N}=Z_{p}+Z_{t}$. The numbers refer to the specific reaction in Ref.[8]. The diagonal full blue line represents the empirical boundary between reactions with no mass-angle correlation (left) and those with (right). To the right of the diagonal red dashed line are reactions which show a minimum at mass-symmetry in the angle-integrated mass distribution. The thin line purple represents the locus of reactions with ${ }^{208} \mathrm{~Pb}$. Examples of $M A D$ are shown in the panels above, with their reaction number. The purple circles and arrow refer to $\mathrm{Cr}+\mathrm{Pb}$ measurements discussed towards the end of the article (adapted from Ref.[8]).

\section{Static Deformation}

The effect of static deformation of the heavy reaction partner on the reaction dynamics has been clearly shown in the dependence of the MAD characteristics with beam energy from below to above the average capture barrier energy B [10]. For deformed nuclei, at energies below B, the reduced Coulomb repulsion for contact on the tips [6] allows capture for elongated (deformation aligned) configurations only, as illustrated schematically on the right side of Fig.4. To the left, experimental mass widths for reactions of ${ }^{48} \mathrm{Ti}$ with a range of heavy nuclei are shown, extending from ${ }^{144} \mathrm{Sm}$ (spherical) through ${ }^{154} \mathrm{Sm},{ }^{174} \mathrm{Yb},{ }^{192} \mathrm{Os}$ (all prolate deformed) to ${ }^{208} \mathrm{~Pb}$ (spherical). For deformed heavy nuclei, large mass widths are found at below-barrier energies $(\mathrm{E} / \mathrm{B}<1)$ corresponding to MAD of type 2 (see Fig.2). 
At $\mathrm{E} / \mathrm{B}>1$, narrowing mass widths show the increasing contribution from more compact equatorial contact configurations, resulting in longer sticking times and mass distributions closer to symmetry.

Already in 1995, interpretation of experimental fission measurements from below to above-barrier led to the suggestion [11] that deformation alignment should be important in SHE synthesis - and indeed, it has been observed that using prolate deformed actinide nuclei, SHE are not created where $\mathrm{E} / \mathrm{B}<1$. This contrasts with reactions of spherical nuclei.

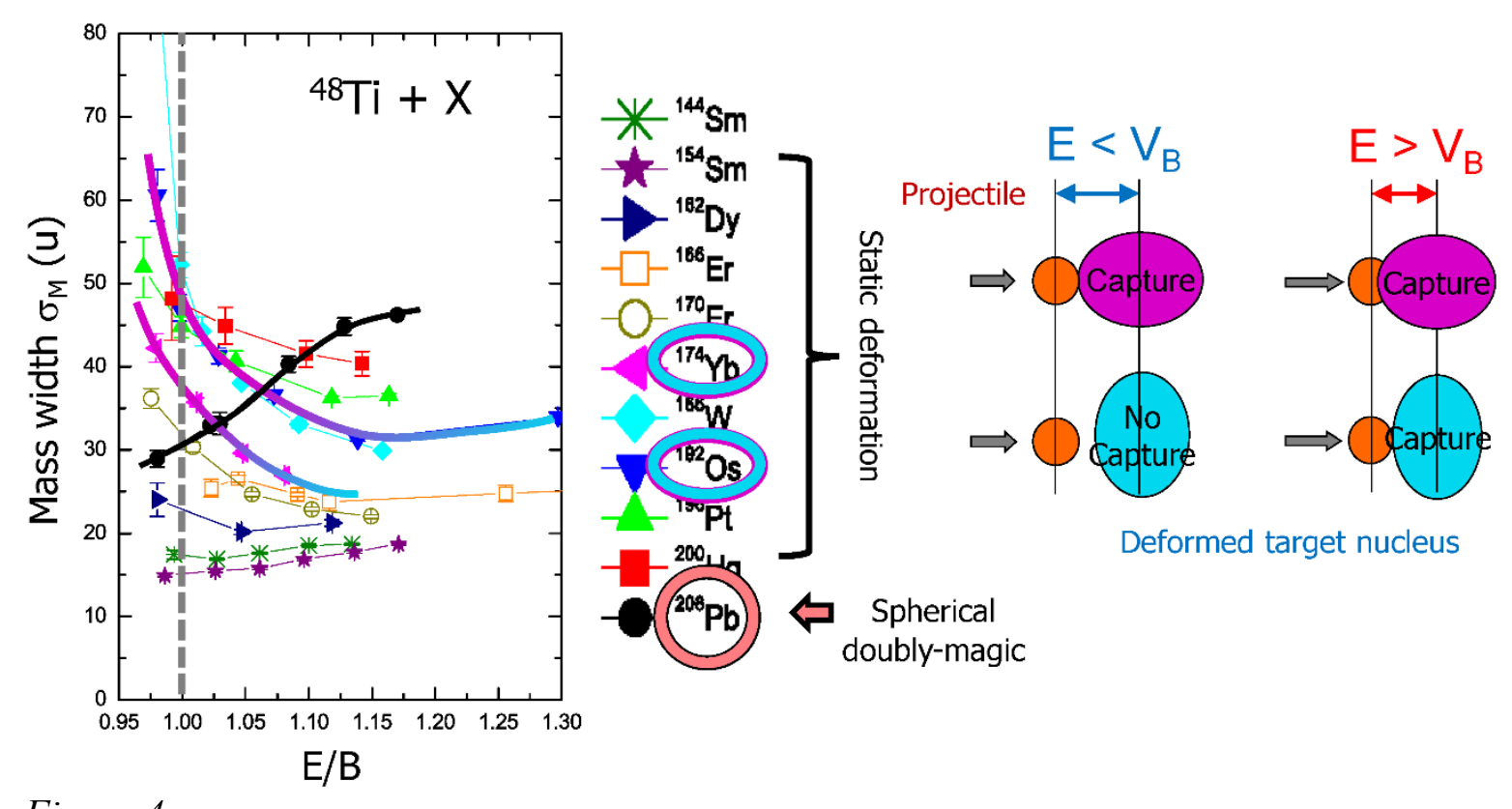

Figure 4

The left panel (adapted from [12]) shows the fragment mass-width (converted from massratio) for the target nuclei indicated. It is plotted as a function of the ratio of the c.m. kinetic energy $\mathrm{E}$ of the ${ }^{48}$ Ti projectile to the average capture barrier energy $\mathrm{B}$. For the reactions of heavy, statically deformed nuclei, the mass width rises rapidly as the energy falls across the barrier. In contrast, the heavy spherical doubly-magic nucleus ${ }^{208} \mathrm{~Pb}$ shows the opposite behaviour (black line). The sketches on the right illustrate that at energies below $\mathrm{B}$, only deformation-aligned collisions result in contact and capture. At $\mathrm{E} / \mathrm{B}>1$, the distance of closest approach is smaller (red arrow), and capture also occurs for more compact configurations, giving longer sticking times, correlated with larger $P_{C N}$.

In reactions of much heavier projectiles with prolate deformed actinide nuclei, such as ${ }^{238} \mathrm{U}$, the difference in observed MAD between the elongated, deformation-aligned contact configurations found below-barrier, and the more compact equatorial collisions is even greater [13]. This is illustrated in Fig.5 for the reaction ${ }^{40} \mathrm{Ca}+{ }^{238} \mathrm{U}$. The sub-barrier measurement shows a MAD of type 1 , with time scale $\sim 5 \mathrm{zs}$. Above-barrier, masssymmetric events become increasingly probable, associated with compact contact configurations and longer sticking times.

Microscopic quantal mean-field TDHF calculations [14] were carried out for this reaction for two extreme orientations: deformation-aligned (axial) and equatorial collisions. The axial collisions had a sticking time typically $6 \mathrm{zs}$, almost independent of angular momentum. Furthermore the Z, N and A of the quasifission fragments from axial collisions showed little variation with angular momentum, excitation energy, or with sticking time, being centred on 82, 122 and 204 respectively. This suggests that the Z,N closed shells around ${ }^{208} \mathrm{~Pb}$ are important in the TDHF calculations. Since they show good agreement with the experimental MAD, this implies that shell effects are playing an important role in quasifission dnamics. In the calculations, equatorial collisions for low impact parameters do not re-separate within $25-40 \mathrm{zs}$, and are taken as resulting in fusion, and subsequent 
fusion-fission. The higher impact parameter equatorial collisions have sticking times and mass-ratios strongly dependent on angular momentum.

For a given sticking time, the equatorial collisions generally experience more massequilibration than the axial collisions, suggesting a special role of the ${ }^{208} \mathrm{~Pb}$ double closed shell in limiting the mass flow to symmetry in the axial collisions. However,

experimentally the mass-asymmetric peaks resulting from axial collision are quite broad, inconsistent with the narrow potential valley near ${ }^{208} \mathrm{~Pb}$. Thus it may be that the effect of the ${ }^{208} \mathrm{~Pb}$ structure on the mass distributions has a dynamical origin, and the mechanism, including the effect of fluctuations, needs more investigation.

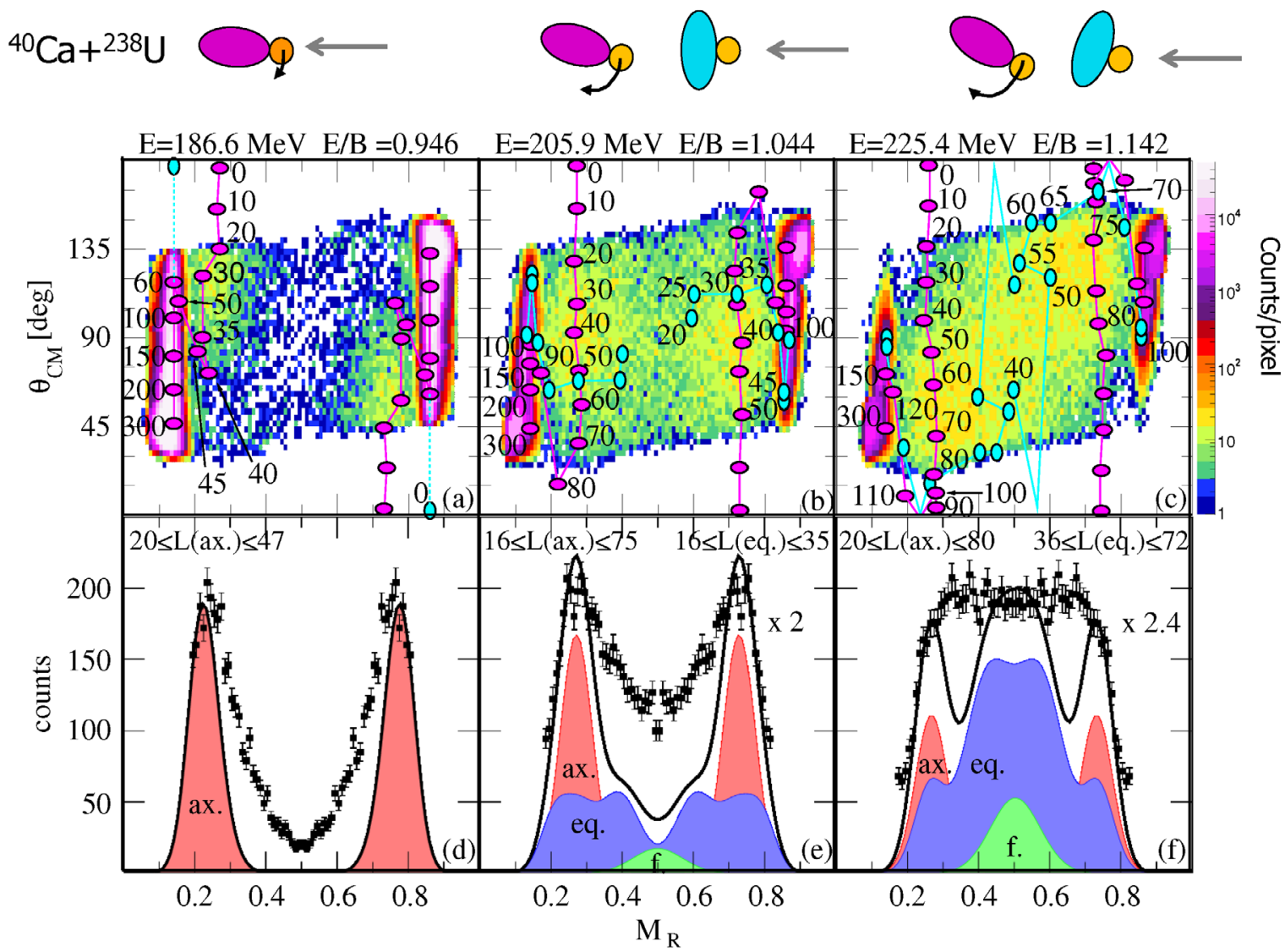

Figure 5

Comparison of experimental MAD (see pixel count colour scale) and mass-ratio spectra (below) with the mass-ratio and angle outcome of TDHF calculations for axial (red ellipses) and equatorial (blue) collisions at the indicated angular momenta next to the symbols [adapted from [14]]. Comparisons from below-barrier $(E / B=0.946)$ to abovebarrier $(E / B=1.142)$ show TDHF agrees well with experiment. Axial collisions have short sticking times and arrested mass-evolution towards symmetry, in contrast with equatorial collisions, which at low angular momenta remain as one nucleus, assumed to eventually undergo mass-symmetric fission (labelled f, and coloured green).

\section{Spherical Magic Nuclei}

Sub-barrier collisions involving heavy (spherical) closed-shell nuclei show contrasting behaviour to those with heavy statically deformed nuclei. This is highlighted in Fig.4, where the mass width for the ${ }^{48} \mathrm{Ti}+{ }^{208} \mathrm{~Pb}$ reaction falls strongly as the beam energy drops, in contrast to the lighter deformed nuclei, where it rises steeply.

To investigate in detail the effect of closed shells in the entrance channel on quasifission probabilities and characteristics, measurements [15] of MAD were made for ${ }^{40,44,48} \mathrm{Ca}$ projectiles bombarding targets of ${ }^{208,204} \mathrm{~Pb}$ (forming ${ }^{248,252} \mathrm{No}$ with $\mathrm{Z}_{\mathrm{C} . \mathrm{N} .}=102$ ), and for ${ }^{48} \mathrm{Ti}$ bombarding ${ }^{200} \mathrm{Hg}\left({ }^{248} \mathrm{No}\right)$ and ${ }^{208} \mathrm{~Pb}\left({ }^{256} \mathrm{Db}\right.$ with $\left.\mathrm{Z}_{\mathrm{C} . \mathrm{N} \text {. }}=104\right)$. Measurements were made a 
few percent below the average fusion barrier energy to give "gentle" collisions with minimal relative velocity at contact.

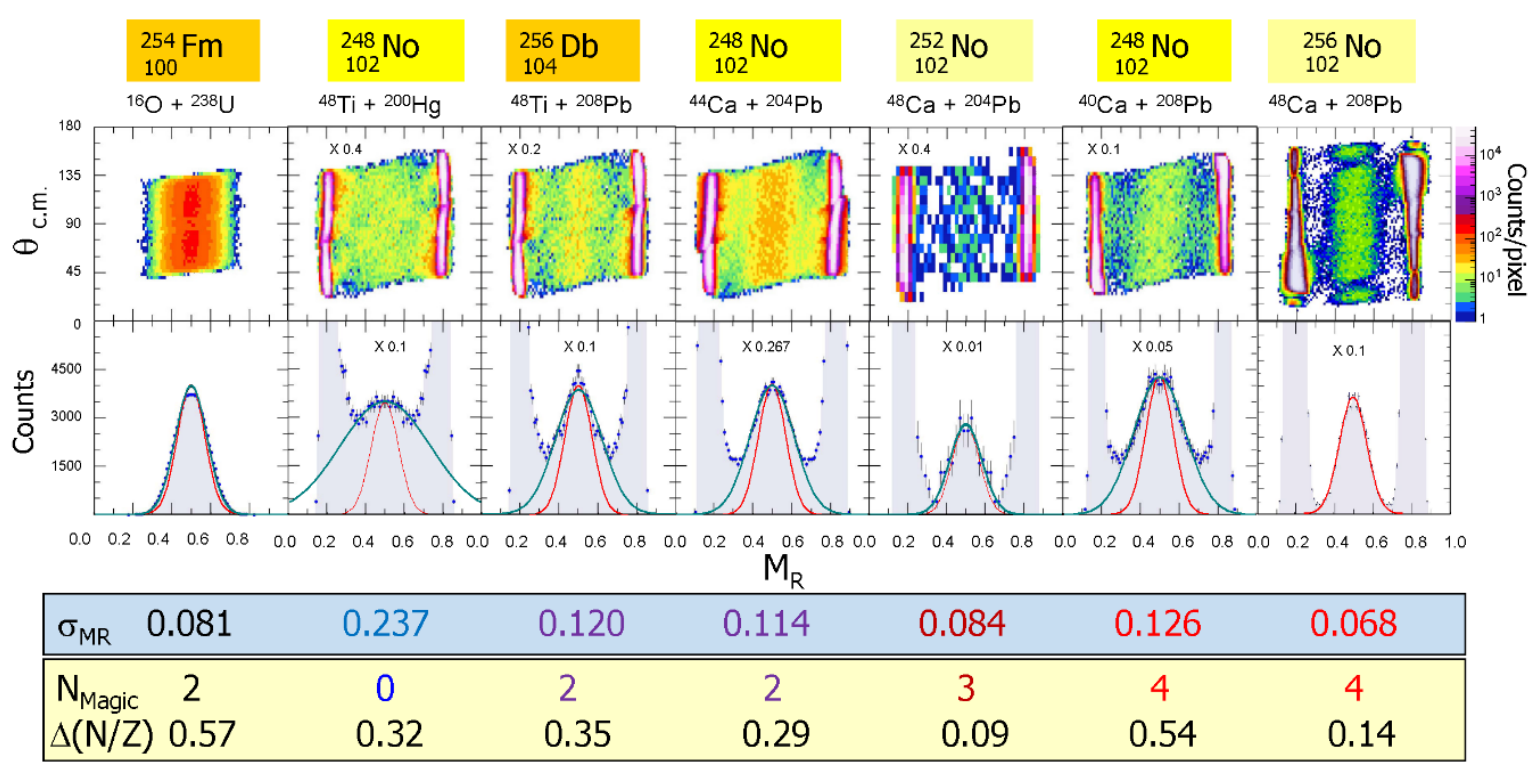

Figure 6

Measured mass-angle distributions for the indicated reactions at $E / B=0.98$ (upper panels), compared to ${ }^{16} \mathrm{O}+{ }^{238} \mathrm{U}$ (left) at an above-barrier energy. In the projected mass ratio spectra for $45^{\circ}<\theta_{\text {c.m. }}<135^{\circ}$ (lower panels) the scale factor multiplies the counts scale on the left. Gaussian functions with $\sigma_{M R}=0.07$ (thin red lines) are shown for reference. Gaussian fits to the region around $M_{R}=0.5$ are shown (turquoise lines), whose standard deviations $\sigma_{M R}$ are tabulated, along with the total number of magic numbers of the projectile and target nuclei $N_{\text {Magic, }}$ and the difference $\Delta(N / Z)$ between the projectile and target nucleus N/Z ratios. Adapted from Ref.[15], with new ANU data for ${ }^{48} \mathrm{Ca}+{ }^{208} \mathrm{~Pb}$.

The MAD and projected $M_{R}$ spectra are shown in Fig.6, together with reference data for the ${ }^{16} \mathrm{O}+{ }^{238} \mathrm{U}$ reaction at an above-barrier energy, which forms ${ }^{254} \mathrm{Fm}$. The standard deviations $\sigma_{M R}$ of the Gaussian fits are tabulated. Despite having similar or identical $\mathrm{Z}_{\mathrm{p}} \mathrm{Z}_{\mathrm{t}}$, and forming similar or identical compound nuclei, values of $\sigma_{M R}$ differ by more than a factor of three, indicating a significant variation in the characteristics and probability of quasifission. Indeed, for the ${ }^{48} \mathrm{Ca}+{ }^{208} \mathrm{~Pb}$ reaction, mass distribution widths appear consistent with a fusion-fission mechanism. To understand this, the correlation of $\sigma_{M R}$ with the sum of the number of magic numbers in the projectile and target nuclei $\mathrm{N}_{\text {Magic }}$ is informative [15]. Large values of $\sigma_{M R}$ are found for no magic numbers, reducing to values expected for fusion-fission for maximal $\mathrm{N}_{\text {Magic }}\left(\right.$ for ${ }^{48} \mathrm{Ca}+{ }^{208} \mathrm{~Pb}$ ). This suggests that reactions involving nuclei having several magic numbers form a compact compound nucleus with higher probability. It seems likely that this is associated with reduced energy dissipation as the two nuclei come together, allowing more compact shapes to be reached. The difference between the N/Z values of the target and projectile nuclei are also shown, denoted by $\Delta(\mathrm{N} / \mathrm{Z})$. The correlation of $\sigma_{M R}$ with $\Delta(\mathrm{N} / \mathrm{Z})$ shows that "magicity" plays its strongest role when the $N / Z$ values of the projectile and target nuclei are well-matched. When this is not the case, transfer reactions even before contact are expected to change the identity of the nuclei, and thus attenuate the entrance-channel magicity, as seen for the ${ }^{40} \mathrm{Ca}+{ }^{208} \mathrm{~Pb}$ reaction [15].

More recent $\mathrm{ANU}$ quasifission results, for the reaction of isotopes of $\mathrm{Cr}$ with $\mathrm{Pb}$, support these conclusions regarding both magicity and N/Z matching. MAD and mass-ratio projections at a sub-barrier energy for each reaction studied are shown in Fig.7. The three reactions on the left all form the same compound nucleus ${ }^{258} \mathrm{Sg}$. 


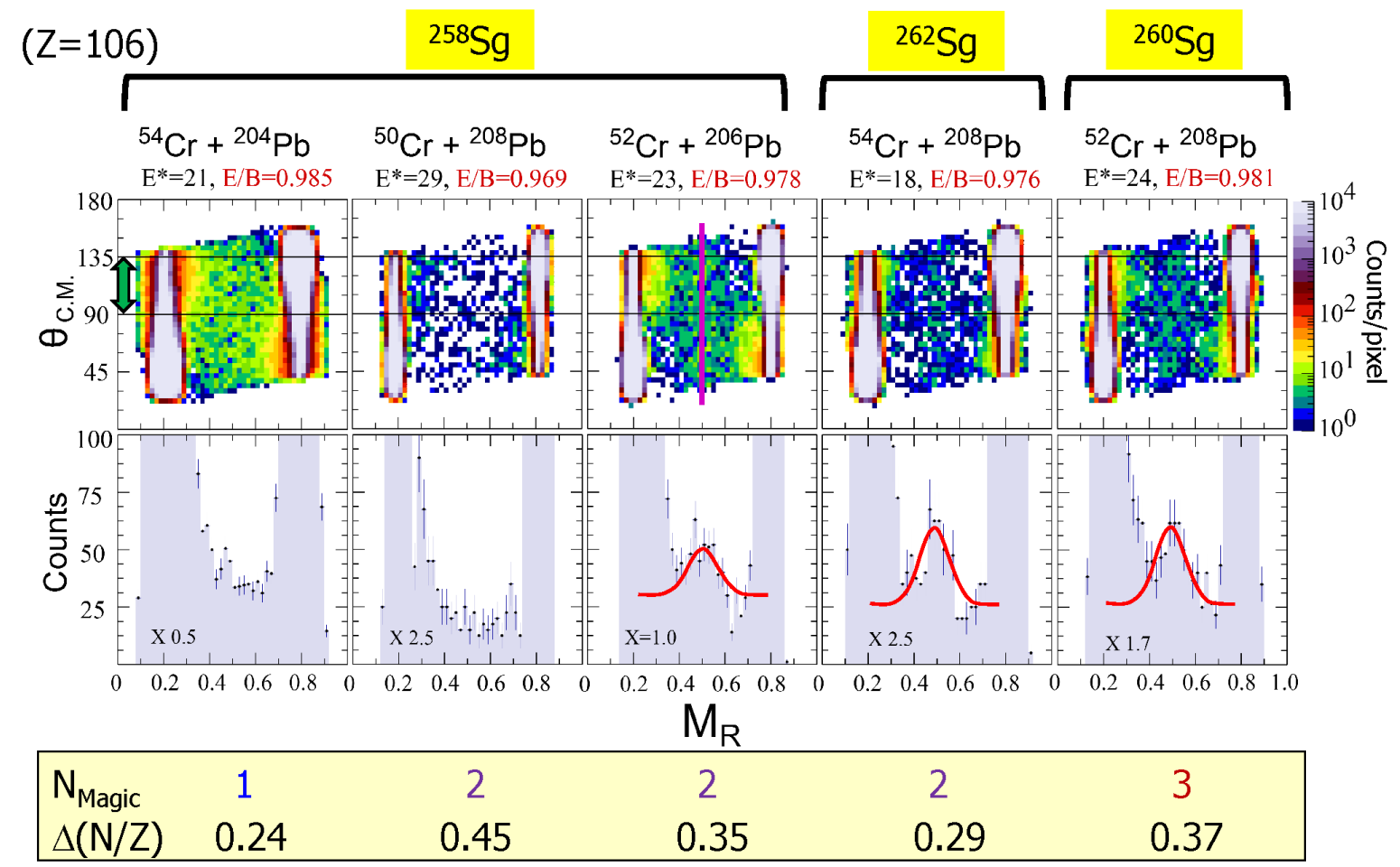

\section{Figure 7}

Experimental MAD and projected mass-ratio spectra $\left(90^{\circ}<\theta_{c . m} .<135^{\circ}\right)$ for sub-barrier (E/B 0.98) collisions of ${ }^{50,52,54} \mathrm{Cr}$ isotopes with ${ }^{204,206,208} \mathrm{~Pb}$, resulting in the low excitation energies $E^{*}$ as indicated. At the bottom of the figure both $N_{\text {Magic, }}$ and $\Delta(N / Z)$ are indicated for each reaction, as in Fig.6. Reactions with more magic numbers and more favourable $\Delta(N / Z)$ display an angle-independent mass-symmetric fission component (purple line for ${ }^{52} \mathrm{Cr}+{ }^{206} \mathrm{~Pb}$ ), suggestive of fusion-fission. The superimposed red Gaussians represent the measured mass-width for the ${ }^{48} \mathrm{Ca}+{ }^{208} \mathrm{~Pb}$ reaction.

The panels are ordered from left to right firstly by the number of magic numbers in the entrance channel $\mathrm{N}_{\text {Magic, }}$ and then by $\Delta(\mathrm{N} / \mathrm{Z})$. The left-most reaction has only a single magic number in the entrance channel, and shows a U-shaped mass distribution, consistent with the MAD1 category. In the representation of systematics in Fig.3, these reactions lie at the position of the right-most purple circle, thus a mass distribution with a minimum in yield at symmetry would indeed be expected. With two magic numbers, the reactions better matched in $N / Z$ - smaller values of $\Delta(N / Z)$ - show a peak at mass-symmetry, associated with an angle-independent ridge in the MAD. This corresponds to very long sticking times, perhaps even fusion-fission. With three magic numbers, but less favourable $\Delta(\mathrm{N} / \mathrm{Z})$, a similar result is observed.

These reactions show the same dependence on magic numbers and N/Z matching as the ${ }^{40,44,48} \mathrm{Ca}+{ }^{204,208} \mathrm{~Pb}$ reactions. However, the transition from a U-shaped mass distribution to a narrow distribution peaked at mass-symmetry, with no evidence for a mass-angle correlation, is a very drastic change in reaction outcome. This is direct evidence of a major bifurcation in reaction trajectories for a given reaction, which is less obvious in the $\mathrm{Ca}+\mathrm{Pb}$ MADs. Fig. 3 indicates that in reactions forming Sg, a MAD of class 3 would be expected for $Z_{\mathrm{p}} \mathrm{Z}_{\mathrm{t}}<1200$ (left-most purple circle), in the absence of the favourable effect of magic numbers. This would correspond to a reaction with an Al projectile or lighter, with around half the atomic number of $\mathrm{Cr}$. The dramatic change in reaction outcome with a small change in neutron number indicates that the observed systematic behaviour at higher beam energies does not necessarily allow prediction of behaviour in near-barrier reactions, where the nuclear structure of the system can play a very significant role (as is also the case in the characteristics of spontaneous and low energy nuclear fission). 
It is clear that both static deformation and spherical magic numbers in the colliding nuclei can play a major role in determining the sticking time in a collision. This ranges from rapid reseparation with only a small change in mass, to sticking together so long that the fission characteristics are those of fusion-fission.

\section{Future Prospects}

To provide a deeper understanding of fusion of heavy nuclei, and of SHE synthesis reactions in particular, measurement of quasifission MADs for a range of projectile and target combinations (projectiles from ${ }^{48} \mathrm{Ca}$ to ${ }^{64} \mathrm{Ni}$, and targets from ${ }^{208} \mathrm{~Pb}$ to ${ }^{249} \mathrm{Cf}$ ) are underway at the Australian National University, in collaboration with researchers from GSI and Mainz. They will add to extensive measurements [16] of mass and total kinetic energy correlations for fragments emerging around $90^{\circ}$ in the centre of mass frame. These new data should provide important information on the time-dependence of the quasifission observables.

The very sudden changes seen in quasifission characteristics, depending on magicity, neutron number, and beam energy will be a severe challenge for models of quasifission and SHE formation to reproduce. And yet it is this level of sensitivity of reaction dynamics to nuclear structure that models must strive to reproduce, to map out the optimum experimental opportunities to create new superheavy elements and isotopes in the future.

\section{Acknowledgments}

Experiments were carried out at the Australian National University Heavy Ion Accelerator Facility. All nuclear reaction dynamics group members are gratefully acknowledged for their contributions to experiments, data analysis, and interpretation.

\section{Funding}

This work has been supported by Australian Research Council Grants DP140101337, DP160101254, DP170102318, DE140100784, FT120100760 and FL110100098, and accelerator operations support from the Australian Federal Government NCRIS program.

\section{References}

[1] B.B. Back et al., Phys. Rev. Lett. 50(1983)818

[2] J. Tōke et al., Nucl. Phys. A440 (1985)327

[3] R. du Rietz et al., Phys. Rev. Lett. 106(2011)052701

[4] D.J. Hinde et al., Phys. Rev. Lett. 89(2002)282701; Phys. Lett. B622(2005)23

[5] A.C. Berriman et al., nature 413(2001)144

[6] M. Dasgupta et al., Annu Rev. Nucl. Part. Sci. 48(1998)401

[7] D.J. Hinde et al., Phys. Rev. C 53(1996)1290

[8] R. du Rietz et al., Phys. Rev. C 88(2013)054618

[9] E. Williams et al., Phys. Rev. C 88(2013)034611

[10] D.J. Hinde et al., Phys. Rev. Lett. 100(2008)202701

[11] D.J. Hinde et al., Phys. Rev. Lett. 74(1995)1295

[12] C.J. Lin et al., Phys. Rev. C 85(2012)014611

[13] D.J. Hinde et al., Phys. Rev. Lett. 101(2008)092701

[14] A. Wakhle et al., Phys. Rev. Lett. 113(2014)182502

[15] C. Simenel et al., Phys. Lett. B710(2012)607

[16] M.G. Itkis et al., Nucl. Phys. A944(2015)204 